\title{
La Voce dei Pazienti Yoga, pilates o cyclette? Forza, diamoci una mossa!
}

P.G.: Ciao a tutti, vi chiedo un'informazione. leri ho fatto una prima lezione di yoga molto dolce e tranquilla. Prima di confermare la mia iscrizione al corso, volevo sapere se qualcuno di voi lo pratica, perché ok che sono fuori forma, ma a piegarmi nelle varie posizioni mi manca l'aria, come se i miei reni ormai giganti mi schiacciassero polmoni e diaframma. Penso sia solo impressione, ma vorrei la certezza di poterlo fare. Mi fate sapere? Grazie mille.

B.S.: lo, oltre ai reni, ho anche il fegato policistico... faccio pilates e se informi l'istruttore ti può dare la variante negli esercizi.

C.M: Lo yoga e pilates fanno molto bene, i loro vantaggi sono innumerevoli sul piano fisico e psichico. Ma al di là del nostro punto di vista è consigliabile che tu ne parli con l'istruttore che con le opportune varianti riuscirà a farti amare ancora di più questa disciplina.

M.R.P.: Cara Pat, io non riesco nemmeno a mettere le scarpe, e per dormire devo assecondare le cisti, correre poi è impossibile.

P.G.: Per me la grossa difficoltà sono i collant.

M.R.P.: Io non li posso portare, mi comprimono. Sono diventata sexy: uso le autoreggenti.

M.V.: Io solo pantaloni con elastici regolabili ovviamente e niente più collant, non sopporto più niente che stringa in vita ormai.

G.V.: Purtroppo sono messa uguale anch'io, per me piegarmi è impossibile, mi manca il respiro, perché le cisti ormai mi arrivano allo stomaco e penso che mi schiaccino i polmoni, almeno questa è la sensazione! E sono già stata operata di ernia addominale!

S.G.: Io faccio yoga con parecchi benefici. Magari informa il tuo maestro, e vedrai che alcune cose potrai farle in maniera diversa.

I.E.: E pilates? Chi di voi lo fa? Mi hanno detto che rinforza la colonna vertebrale.

S.G.: Faccio pure quello.

A.G.: Ciao, io ho praticato yoga per molti anni, quando ancora le cisti erano di moderata dimensione. Ho riprovato due anni fa e non riuscivo più: i piegamenti che sono riuscita a fare mi hanno provocato anche la febbre... probabilmente avevo "disturbato" le mi cisti!

A.Z.: lo faccio yoga, ma non ho le cisti grosse.

P.R.: Pat, parlane con l'insegnante di yoga. Sapendo dei tuoi problemi, magari ti fa fare delle varianti per certe posizioni in cui fai fatica. lo, quando lo facevo, le avevo parlato dei miei problemi di pressione e non mi faceva fare le posizioni rovesciate tipo candela, perché diceva che non erano consigliate.

C.B.: Forse la cosa migliore è fare un po' di cyclette a casa e camminate di un'ora a passo veloce, perché così si evitano traumi che ci affaticano a causa dei reni ingrossati.

P.G.: Il mio nefrologo mi ha consigliato di fare un corso senza compressione addominale. E lo yoga, tra tira e piega, comprime parecchio...

M.O.: I miei reni non sono troppo grossi, quindi faccio un'ora quattro mattine la settimana. Pilates, ginnastica posturale, step e stretch\&tone, tutto con la musica. Si lavora molto di gambe, braccia, colonna e addominali. E comunque se si ha difficoltà con gli addominali non è obbligatorio fare gli esercizi, però il movimento fa bene.

R.G.: Le mie cisti sono massimo di $4 \mathrm{~cm}$, quindi non sono poi così grandi. Sono sovrappeso di soli $6 \mathrm{~kg}$ anche per colpa dell'ipotiroidismo. Nonostante tutto, indosso pantaloni solo con elastico in vita, non metto collant e se le scarpe sono con cinturino o stringhe, me le allaccia mio marito. Riguardo allo sport, il mio nefrologo dice che si può fare solo ginnastica dolce.

S.G.: Io ho numerose cisti intorno ai $9 \mathrm{~cm}$ di diametro. Lo sport è indicatissimo. Bisogna fare attenzione solo a non fare sport di contatto e tutto il resto con moderazione. Aiuta non solo per il corpo, ma soprattutto per la mente. Lo yoga poi ha numerose varianti, qualcuna è molto dinamica, altre lo sono meno e aiutano a controllare la respirazione.

M.G.: lo ho dovuto smettere a causa dell'addome ingombrante, ma se non ti sforzi troppo, penso che lo possa fare. Decidi tu se vale la pena, se a certi esercizi devi rinunciare...

A.B.: lo faccio pilates e il mio nefrologo mi ha detto che fa bene. Certo, non riesco a fare tutto e quando porto le ginocchia al petto sento la pancia maledetta che ingombra, ma sapeste quanto mi sento bene dopo...

A.G.: Scusate se mi intrometto in un discorso tra donne... solo gli sport di contatto vanno evitati e chiaramente il motocross e affini, compresa la mountain bike fuoristrada, ma solo perché si può cadere e farsi male Lo yoga si può fare, cosi come il pilates e l'aerobica o la zumba. Anche il ciclismo su strada con moderazione. Insomma, lasciamoci andare e stiamo in forma... lo sport e l'attività fisica aiutano a tenere la pressione arteriosa sotto controllo. Non fissiamoci troppo.

Published online: December 14, 2016 